\title{
VRE and VSE Bacteremia Outcomes in the Era of Effective VRE Therapy: A Systematic Review and Meta-analysis
}

\author{
Chatura Prematunge, MSc; ${ }^{1}$ Colin MacDougall, MSc; Jennie Johnstone, MD, PhD; ${ }^{1,2,3}$ Kwaku Adomako, MSc; $^{1}$ \\ Freda Lam, MPH; ${ }^{1}$ Jennifer Robertson, PhD; ${ }^{1}$ Gary Garber, $\mathrm{MD}^{1,3,4,5}$
}

\begin{abstract}
BACKGROUND. Prior data suggest that vancomycin-resistant Enterococcus (VRE) bacteremia is associated with worse outcomes than vancomycin-sensitive Enterococcus (VSE) bacteremia. However, many studies evaluating such outcomes were conducted prior to the availability of effective VRE therapies.
\end{abstract}

овлестіVе. To systematically review VRE and VSE bacteremia outcomes among hospital patients in the era of effective VRE therapy.

MEтноDS. Electronic databases and grey literature published between January 1997 and December 2014 were searched to identify all primary research studies comparing outcomes of VRE and VSE bacteremias among hospital patients, following the availability of effective VRE therapies. The primary outcome was all-cause, in-hospital mortality, while total hospital length of stay (LOS) was a secondary outcome. All meta-analyses were conducted in Review Manager 5.3 using random-effects, inverse variance modeling.

RESUlts. Among all the studies reviewed, 12 cohort studies and 1 case control study met inclusion criteria. Similar study designs were combined in meta-analyses for mortality and LOS. VRE bacteremia was associated with increased mortality compared with VSE bacteremia among cohort studies (odds ratio $[\mathrm{OR}], 1.80 ; 95 \%$ confidence interval $[\mathrm{CI}], 1.38-2.35 ; \mathrm{I}^{2}=0 \% ; \mathrm{n}=11$ ); the case-control study estimate was similar, but not significant (OR, 1.93; 95\% CI, 0.97-3.82). LOS was greater for VRE bacteremia patients than for VSE bacteremia patients (mean difference, 5.01 days; $95 \% \mathrm{CI}, 0.58-9.44] ; \mathrm{I}^{2}=0 \% ; n=5$ ).

CONCLUSions. Despite the availability of effective VRE therapy, VRE bacteremia remains associated with an increased risk of in-hospital mortality and LOS when compared to VSE bacteremia.

Infect. Control Hosp. Epidemiol. 2016;37(1):26-35

Enterococcus spp. are typically commensal organisms, common in the human gastrointestinal tract, ${ }^{1,2}$ but in some circumstances can cause serious infections including bacteremia, particularly among hospitalized patients with underlying comorbid conditions. ${ }^{1,2}$ Since its discovery in 1988, vancomycin-resistant enterococci (VRE) have emerged as important nosocomial pathogens and are occurring with increasing frequency due to widespread use of antibiotics, prolonged hospitalizations, and increased intensive care unit (ICU) admissions, especially among patients with malignant health conditions. ${ }^{1-3}$ In Canada, the incidence of VRE infections has risen to 0.5 infections per 1,000 admissions, a 6-fold increase in recent years. ${ }^{4}$ Similarly in the United States, hospitalizations with VRE infection reached 0.6 per 1,000 admissions by $2006{ }^{3}$
Whether outcomes associated with VRE bacteremia are worse than those associated with vancomycin-sensitive enterococci (VSE) bacteremia remains unclear. Two prior systematic reviews have compared outcomes of VRE bacteremia VSE bacteremia; both found VRE bacteremia to be associated with an increased risk of mortality when compared to VSE bacteremia (relative risk [RR], 2.38; 95\% confidence interval $[\mathrm{CI}], 2.13-2.66 ;^{5}$ odds ratio $[\mathrm{OR}], 2.52 ; 95 \% \mathrm{CI}$, $\left.1.87-3.39^{6}\right)$. However, both of these systematic reviews included studies conducted prior to the availability of effective VRE therapies. ${ }^{56}$ Since late 1999, a number of antibiotic drugs have been licensed as treatment for VRE bacteremia by the US Food and Drug Administration (FDA), Health Canada, and other national approval agencies. ${ }^{1,2,7}$ Quinupristindalfopristin was approved in 1999, followed by linezolid

Affiliations: 1. Infection Prevention and Control, Public Health Ontario, Toronto, Ontario, Canada; 2. St. Joseph's Health Centre, Toronto, Ontario, Canada; 3. Department of Medicine, University of Toronto, Toronto, Ontario, Canada; 4. Department of Medicine, University of Ottawa, Ottawa, Ontario, Canada; 5. Ottawa Hospital Research Institute, Ottawa, Ontario, Canada.

PREVIOUS PRESENTATION. The preliminary findings of this systematic review and meta-analysis were presented at Association of Medical Microbiology and Immunology (AMMI) Annual Conference, Quebec, Canada, in April 2015 as an oral presentation.

Received April 30, 2015; accepted August 30, 2015; electronically published October 5, 2015

(C) 2015 by The Society for Healthcare Epidemiology of America. All rights reserved. This is an Open Access article, distributed under the terms of the Creative Commons Attribution license (http://creativecommons.org/licenses/ by/3.0/), which permits unrestricted re-use, distribution, and reproduction in any medium, provided the original work is properly cited. All rights reserved. 0899-823X/2016/3701-0006. DOI: 10.1017/ice.2015.228 
in $2000 .^{7}$ In 2003, daptomycin was formally licensed for complicated skin and soft tissue VRE infections, but it is frequently used as an off-label therapy for VRE bacteremia. ${ }^{8}$

Thus, understanding whether VRE bacteremia-associated outcomes are different from those of VSE bacteremia, since the emergence of effective VRE therapy, is critically important to help inform future VRE infection control recommendations. To this end, we performed a systematic review and meta-analysis of studies comparing outcomes of patients with either VRE or VSE bacteremia, when patients with VRE bacteremia were treated with effective VRE therapy.

\section{METHODS}

All methods including literature searches, study selection, data collection, and quantitative analysis processes were developed $a$ priori and were reported according to the Preferred Reporting Items for Systematic Reviews and Meta-Analyses (PRISMA) guidelines and the Cochrane Handbook for Systematic Reviews of Intervention. ${ }^{9,10}$

\section{Search Methodology and Data Sources}

The Public Health Ontario (PHO) Library Services department assisted with the development and implementation of search strategies for electronic databases, as well as with the retrieval of full-text articles. Medline, Embase, CINAHL, ProQuest dissertations and theses, and the Cochrane Central Register of Controlled Trials (CENTRAL) databases were searched from January 1997 to December 2014. A sample search strategy is provided in Supplemental Table 1. Websites of infection control authorities and proceedings from infection control conferences held within the most recent 5 years (ie, January 1, 2010, to January 1,2015) were searched as outlined in Supplemental Table 2. Conference proceedings prior to 2010 were not considered because we assumed that valuable data contained within such abstracts had become available in peer-reviewed literature. Additionally, the reference lists of all relevant publications were hand searched to identify additional citations.

\section{Study Inclusion Criteria}

The study inclusion criteria for the review were randomized controlled trials (RCTs), cohort studies, case-control studies, and cross-sectional studies, sampling adult ( $\geq 18$ years of age) and/or pediatric ( $<18$ years of age) hospital patients, diagnosed with VRE bacteremia and treated with effective VRE therapy, alongside VSE bacteremia patient comparators, and reporting on various mortality and morbidity outcomes. The primary outcome of interest was all-cause in-hospital mortality. Secondary outcomes were bacteremia-attributable mortality, total hospital length of stay (LOS), total intensive care unit (ICU) LOS, post-VRE/VSE bacteremia diagnosis hospital LOS, and post-VRE/VSE bacteremia diagnosis ICU LOS. Effective VRE therapies were defined as quinupristindalfopristin, linezolid, daptomycin, tigecycline, teicoplanin, and telavancin for treating any part of the illness. ${ }^{1,2,7,8}$ Penicillin, ampicillin, amikacin, streptomycin, chloramphenicol, doxycycline, rifampin, imipenem-cilastatin, and nitrofurantoin were not considered effective VRE treatments. ${ }^{11}$

To capture standard, off-label, and compassionate study use of effective VRE treatment(s), literature published after January 1997 was considered. Studies analyzing data collected between January 1997 and January 2000 were excluded if the antibiotics used for the treatment of VRE bacteremia patients were not reported or could not be obtained by contacting study authors. Studies conducted after January 2000 were assumed to have administered effective VRE treatment(s) and were included in the review.

Narrative reviews, case series, case reports, and commentaries were excluded. Only the most recent peer-reviewed publication was included when multiple reports using the same study data existed. We limited our review to English language articles. ${ }^{12}$

\section{Study Selection and Data Extraction}

Titles and abstracts of articles captured by literature searches were independently screened in duplicate by two reviewers ( $\mathrm{CP}$ and $\mathrm{CM})$. Articles flagged for full-text review by either reviewer were included in the full-text review, and the full-text review process was duplicated and independently completed by the same reviewers. Inter-rater reliability following full-text review was calculated using Cohens Kappa statistic and any disagreements on study inclusion were resolved via arbitration by a third reviewer (JJ).

\section{Quality Assessment}

Data extraction and quality assessment for included studies were performed in duplicate (by $\mathrm{CP}$ and $\mathrm{CM}$ ). An electronic data extraction template was developed, pilot tested, and refined prior to the initiation of data extraction. The extracted data elements included study design, sample size, study period, study setting, study population, patient type, study location, Enterococcus spp., VRE/VSE bacteremia definition, VRE therapy administered, and number of VRE and VSE bacteremia patients with the above stated outcome(s) of interest along with associated effect estimates and confidence intervals. Whenever required information was not reported, attempts were made to contact the first and/or corresponding authors to obtain missing information; after 2 attempts, authors were considered unresponsive. Data requests were limited to missing information on administered VRE treatment type(s), primary outcomes, and any secondary outcomes reported within the primary study.

Study quality was assessed using the Newcastle-Ottawa Scale (NOS) scale or Cochrane risk of bias tool. The NOS was used to establish quality of evidence within non-randomized cohort or case control studies, via a 9-star system. ${ }^{13}$ A study awarded a greater number of stars is considered to be of higher methodological study quality. ${ }^{13}$ Although we did not 
anticipate finding any RCTs, the Cochrane risk of bias tool was assigned to assess RCT study quality in the event an RCT meeting inclusion criteria was discovered. ${ }^{10}$

\section{Data Analysis}

Outcome effect measures for each study were calculated using numbers of patients with VRE and VSE bacteremia with the outcome(s) of interest. We pooled studies of the same study design via inverse variance method and random effects modeling in Review Manager 5.3; summary effect measures are reported as odds ratios (OR) and $95 \%$ confidence intervals (CI) for mortality, and mean difference and standard deviation (SD) are reported for continuous LOS outcomes. When the median and interquartile ranges (IQR) were reported, the median was assumed to reflect the mean, ${ }^{14}$ and IQR was assumed to be $1.35 \mathrm{SD} .{ }^{10}$ Statistical heterogeneity was assessed using the $\mathrm{I}^{2}$ statistic, and VRE and VSE bacteremia outcomes were further explored via planned subgroup analyses of the following patient populations: (1) adult versus pediatric patients, (2) immunocompromised versus varied immune status patients, ICU versus non-ICU admissions, (3) multicenter versus single study site, and low versus moderateto-high study quality for included cohort studies. ${ }^{10}$ Publication bias was examined via the visual interpretation of funnel plot symmetry and limited to the mortality outcomes. ${ }^{10}$

\section{Role of the Funding Source}

The design, conduct, and reporting for this systematic review and meta-analysis was funded by the Ontario Agency for Health Protection and Promotion (Public Health Ontario).

\section{RES ULTS}

\section{Literature Search}

The literature searches identified 4,878 citations; among these, 155 citations were chosen for full-text review, and 20 studies were determined to meet our inclusion criteria (Figure 1). Of these, 1 study did not indicate a study period and 5 studies reported study periods between January 1997 and January 2000 and required confirmation of VRE therapies within each study. Corresponding authors were contacted, but administered VRE therapy information could not be obtained and all 7 studies were excluded from the review. Excluded study details are provided in Supplemental Table 3. Therefore, 13 studies were included in the systematic review.

\section{Description of Studies}

The study characteristics of all included studies are outlined in Table 1. All were observational and retrospective studies, 12 of which were conducted between January 2000 and December 2011, following the formal regulatory approval of the first effective VRE therapy. ${ }^{15-26}$ The study by da Silva et $\mathrm{al}^{27}$ reported a study period between September 1998 and
December 2008. However, these authors confirmed that all patients with VRE bacteremia were diagnosed after January 2000. Billington et $\mathrm{al}^{16}$ sampled all residents within a Canadian health zone who developed enterococcal bloodstream infections. We contacted these authors to obtain mortality and LOS information for study participants. In addition, 8 studies exclusively sampled adult patients within tertiary care hospital settings, ${ }^{15,17-19,21,22,24,26}$ and 4 of these studies were limited to immunocompromised patient populations ${ }^{19,22,25,26}$ such as hematopoietic stem cell transplant patients or chemotherapy recipients.

All included studies defined patients with at least 1 VRE- or VSE-positive blood culture to be cases of bacteremia. ${ }^{15-27}$ Both E. faecalis and E. faecium were captured in 12 study samples, ${ }^{15-25,27}$ but the study by Yoo et $\mathrm{al}^{26}$ only included E. faecium infections. Outcome data for 2,575 bacteremias, specifically 1,863 VSE and 712 VRE bacteremia cases, were identified in our literature review.

\section{Outcomes}

Of the reviewed studies, 12 studies were cohort studies and 1 was a case control study. When in-hospital mortality from the cohort studies were combined using unadjusted analysis, VRE bacteremia was associated with an increased risk of in-hospital death when compared to VSE bacteremia with no heterogeneity (OR, 1.80; 95\% CI, 1.40-2.32; $\mathrm{I}^{2}=0 \% ; \mathrm{n}=12$ ) (Figure 2). The single case-control study did not report a statistically significant increase in risk of VRE bacteremia death when compared with VSE bacteremia in an unadjusted analysis (OR 1.93; 95\% CI, $0.97-3.82)^{24}$; adjusted analyses were not reported.

Of the 12 cohort studies, 5 reported adjusted analyses for in-hospital mortality risk, ${ }^{15,16,18,19,21}$ and 2 studies found VRE bacteremia to be associated with adjusted mortality. ${ }^{18,19}$ Cheah et $\mathrm{al}^{18}$ adjusted for prior ICU admission, comorbidities measured by the Charlson Comorbidity Index, Enterococcus sp., additional non-enterococcal infections, time to effective therapy, and VRE bacteremia (OR, 1.21; 95\% CI, 0.53-2.79]) via logistic regression analysis. Cho et $\mathrm{al}^{19}$ adjusted for severity of illness using Simplified Acute Physiology Index, length of hospitalization, and vancomycin resistance (hazard ratio [HR], 0.75; 95\% CI, 0.24-2.36) via Cox proportional hazards modeling. VRE bacteremia was not included in the final models of the remaining 3 studies reporting adjusted mortality. ${ }^{15,16,21}$

The study by Cho et al was the only study to report on VRE/ VSE bacteremia-attributable mortality, which was defined as death within 7 days of bacteremia when no other cause could be identified. There was no significant difference in attributable mortality risk between VRE and VSE bacteremia patients in the unadjusted analysis (6 of 24 patients with VRE bacteremia vs 15 of 67 patients with VSE bacteremia; OR, 1.15; $95 \%$ CI, 0.39-3.43). ${ }^{19}$

Total hospital LOS data were reported within 6 studies. Data reported by Butler et $\mathrm{al}^{17}$ and Cheah et $\mathrm{al},{ }^{18}$ and data obtained by contacting authors of Billington et al, ${ }^{16}$ da Silva et al, ${ }^{27}$ and 


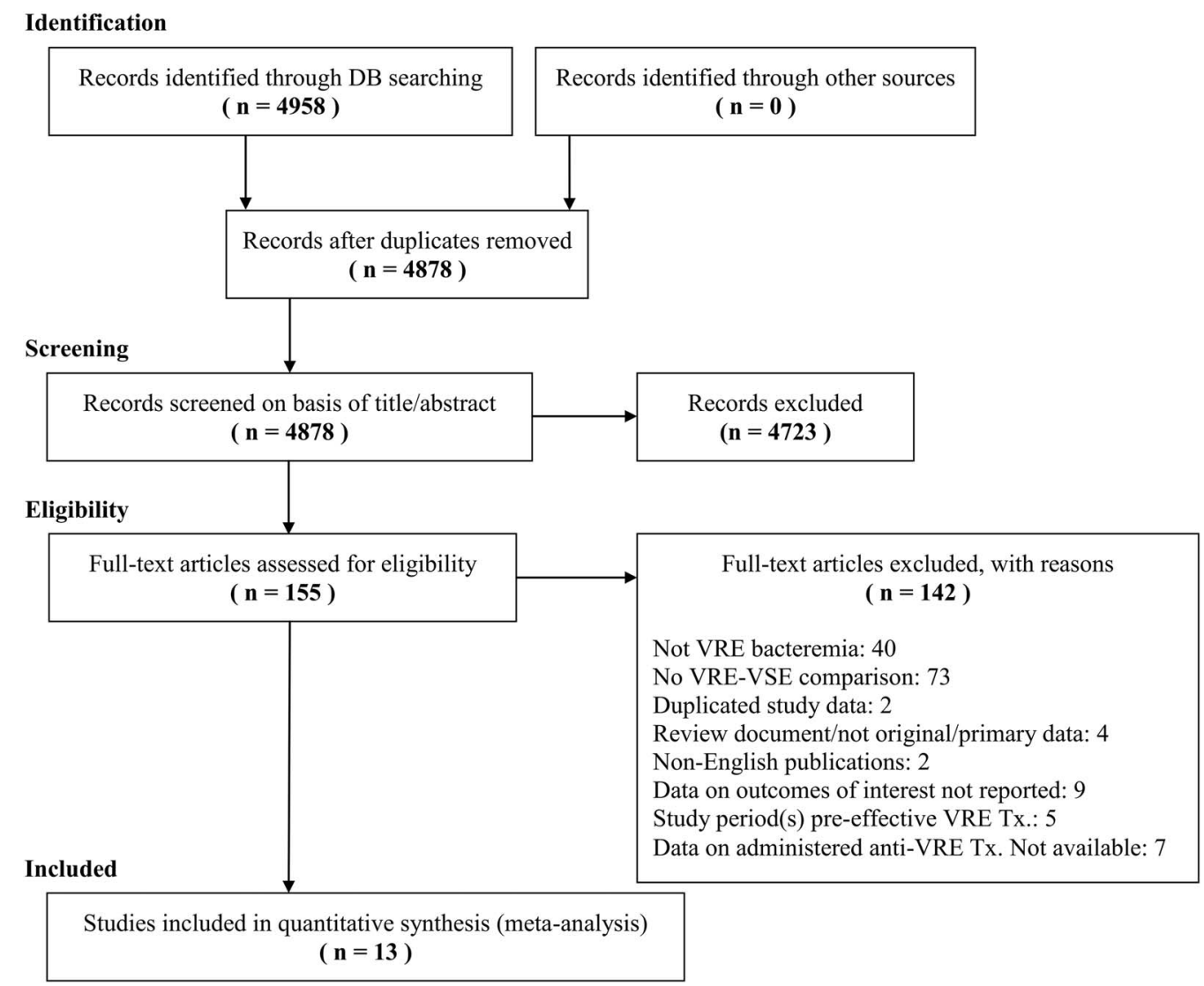

FIGURE 1. Preferred Reporting Items for Systematic Reviews and Meta-Analysis (PRISMA) flowchart of the literature search and study selection.

TA B LE 1. Characteristics of Studies Included in Systematic Review and Meta-Analysis

\begin{tabular}{|c|c|c|c|c|c|}
\hline Study & Study Period & Location & Patient Population & \multicolumn{2}{|c|}{ Sample Size, No. } \\
\hline Bar et al, $2006^{15}$ & Nov 2000-Dec 2002 & Richmond, VA USA & Adult & 17 & 33 \\
\hline Billington et al, $2014^{16}$ & $2000-2008^{\mathrm{a}}$ & Calgary, Canada & Mixed & 27 & 640 \\
\hline Butler et al, $2010^{17}$ & Jan 2002-Dec 2003 & St Louis, MO USA & Adult, Non-surgical, $>2$ days LOS & 94 & 182 \\
\hline da Silva et al, $2014^{27}$ & Sep 1998-Dec 2008 & Sao Jose do Rio Preto, Brazil & Mixed & $30^{\mathrm{c}}$ & $273^{\mathrm{c}}$ \\
\hline Haas et al, $2010^{20}$ & $2001-2006^{\mathrm{a}}$ & Philadelphia, PA USA & Pediatrics & 39 & 300 \\
\hline Marschall et al, $2013^{21}$ & Jan 2006-Dec 2006 & St. Louis, MO USA & Adult, CVC associated bacteremias & 67 & 39 \\
\hline Mikulska et al, $2012^{22}$ & $2004-2011^{\mathrm{a}}$ & Genoa, Italy & Adult, allogeneic HSCT & 9 & 58 \\
\hline Mohr et al, $2009^{23}$ & Jan 2000-Dec 2009 & 58 sites, USA & Mixed, ${ }^{\mathrm{b}}$ dap Tx. & 151 & 211 \\
\hline Vydra et al, $2012^{25}$ & Jan 2004-Dec 2008 & Minneapolis, MN USA & Mixed, HSCT & 50 & 43 \\
\hline
\end{tabular}

NOTE. LOS, length of stay; HSCT, hematopoietic stem cell transplantation; CHEMO, chemotherapy; dap Tx, daptomycin treatment; CVC, central venous catheter; SCT, stem cell transplantation.

${ }^{\mathrm{a}}$ Months not reported.

${ }^{\mathrm{b}}$ Assumed to be mixed, unconfirmed due to demographics being reported as $\leq 30$ years of age.

${ }^{\mathrm{c}}$ Data obtained by contacting study authors.

${ }^{\mathrm{d}} \mathrm{A}$ total of 8 VRE patients received VRE therapies and were included in the review. 


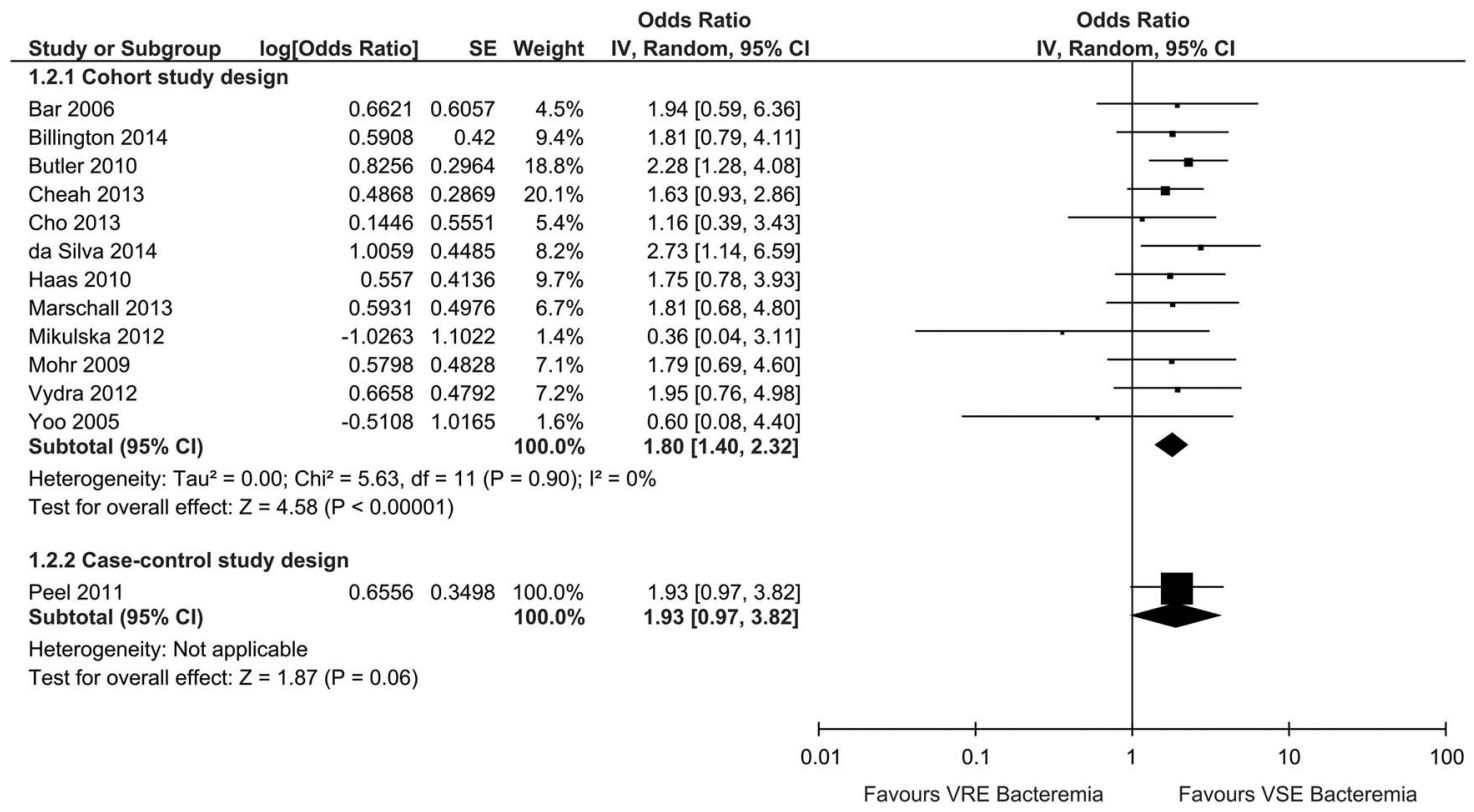

FIGURE 2. VRE and VSE bacteremia unadjusted in-hospital mortality risk by study design. Results of included studies for VRE and VSE bacteremia unadjusted in-hospital mortality risk stratified by study design. Abbreviations: 95\% CI, 95\% confidence interval; SE, standard error; IV, random, inverse-variance, random-effects method.

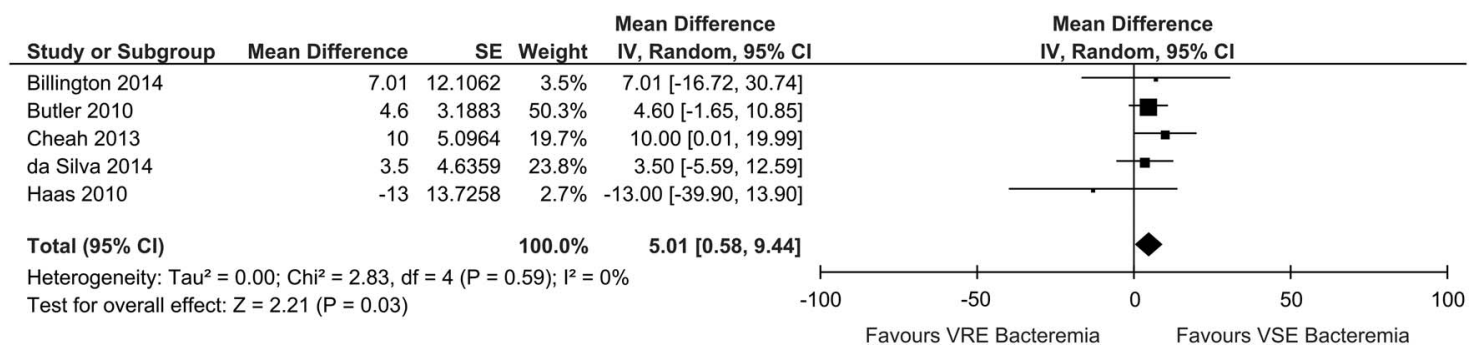

FIGURE 3. VRE and VSE bacteremia total hospital LOS mean difference. Results of studies reporting on VRE and VSE bacteremia total hospital LOS. Abbreviations: LOS, length of stay; 95\% CI, 95\% confidence interval; SE, standard error; IV, random, inverse-variance, random-effects method.

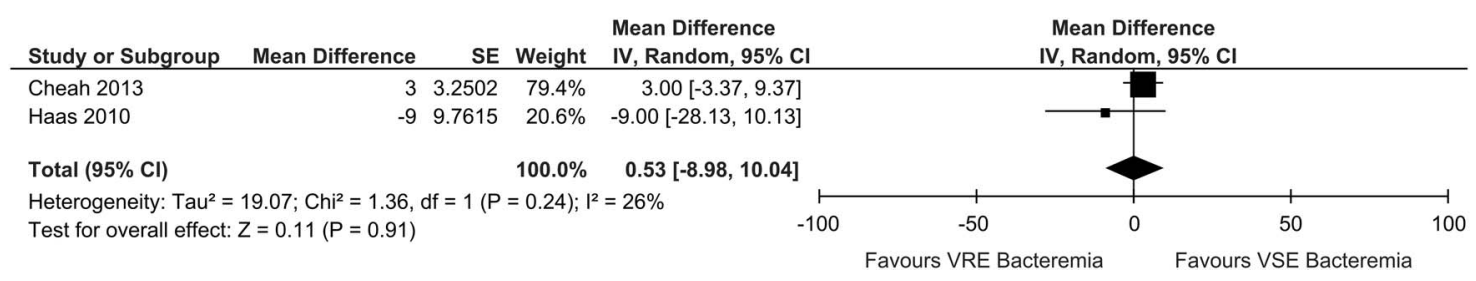

FIGURE 4. VRE and VSE post-bacteremia total hospital LOS mean difference. Results of studies reporting on VRE and VSE post-bacteremia hospital LOS. Abbreviations: LOS, length of stay; 95\% CI, 95\% confidence interval; SE, standard error; IV, random, inverse-variance, random-effects method.

Haas et $\mathrm{al}^{20}$ were pooled; VRE bacteremia was associated with a longer LOS than VSE bacteremia (mean difference, 5.01; 95\% CI, 0.58-9.44; $\mathrm{I}^{2}=0 \% ; \mathrm{n}=5$ ) (Figure 3). Data from Cho et al were excluded because they defined LOS as the number of days from hospital admission to the development of clinically significant bacteremia, which is different from the LOS definition used in our review. Data from Yoo et $\mathrm{al}^{26}$ were excluded because their LOS estimates combined patients treated with effective and noneffective VRE therapy. 
Post-bacteremia LOS data reported by Cheah et al and Haas et al were also pooled via a meta-analysis. There was no significant difference in LOS following a VRE bacteremia compared with VSE bacteremia (mean difference, 0.53 [95\% CI -8.98, 10.04]; $\mathrm{I}^{2}=26 \% ; \mathrm{n}=2$ ) (Figure 4). Yoo et al also reported on post-bacteremia LOS, but data were omitted because estimates combined patients treated with both effective and noneffective VRE therapy.

\section{Subgroup Analyses}

No significant interactions were detected between any of the subgroups we had planned to analyze for in-hospital mortality including age (pediatric patients [OR, 1.62; 95\% CI, 1.18-2.22] vs adult [OR, $1.93 ; 95 \% \mathrm{CI}, 0.89-4.18]$; interaction $P=.68$ ), immune status (immunocompromised patients [OR, 1.24; 95\% CI, 0.65-2.35] vs varied immune status [OR, $1.93 ; 95 \% \mathrm{CI}, 1.47-2.54]$; interaction $P=.21$ ), study site (single center studies [OR, 1.85; 95\% CI, 1.37-2.50] vs multicenter studies [OR, 1.70; 95\% CI, 1.12-2.58]; interaction $P=.75$ ) and study quality (low-quality studies [OR, 0.36; 95\% CI, 0.04-3.11] vs moderate- to high-quality studies [OR, 1.84; 95\% CI, 1.43-2.37]; interaction $P=.14$ ) (Figure 5). The planned subgroup analysis for ICU stay was not performed due to unavailable data.

Age was not found to significantly influence total hospital LOS by subgroup analysis (pediatric patients [OR -13.00 ; $95 \%$ CI, $-39.90-13.90$ ] vs adult [OR, 6.12; 95\% CI, 0.82-11.42]; interaction $P=.17$ ) (Figure 6). The remaining LOS subgroup analyses could not be performed due to a lack of studies in each companion subgroup. No significant interaction was detected in the subgroup analysis of post-bacteremia LOS by age (pediatric patients [OR, $-9.0 ; 95 \% \mathrm{CI},-28.13-10.13$ ] vs adult [OR, 3.0; 95\% CI -3.37-9.37]; interaction $P=.24$ ) (Figure 4).

\section{Study Quality}

Study quality ratings based on NOS criteria are presented in Table 2. Of the 13 studies reviewed, 12 were of moderate to high study quality, with the most frequent number of stars awarded per study being 6 or 7. Among all studies, patients with VRE and VSE bacteremia were selected from the same hospital population, and bacteremia diagnosis was confirmed by patient chart reviews or microbiology reports.

\section{Publication Bias}

The asymmetrical funnel plot indicates that the review's in-hospital mortality estimates may be subject to publication bias (Figure 7).

\section{DISC USSION}

In this systematic review and meta-analysis, we found that since the advent of effective VRE therapy, there remains an increased risk of in-hospital mortality associated with VRE bacteremia compared with VSE bacteremia. The mortality summary estimate demonstrated lack of heterogeneity across studies and no significant influence on the point estimate by age, immune status, study site(s), or study quality. VRE bacteremia was also associated with increased total hospital LOS and post-bacteremia LOS with no heterogeneity. The post-bacteremia LOS estimate was not statistically significant, which may have been due to lack of statistical power influenced by the small number of studies reporting on post bacteremia LOS outcomes.

Our finding, that there is an increased risk of mortality and LOS associated with VRE bacteremia when compared to VSE bacteremia, is consistent with 2 previous systematic reviews. ${ }^{5,6}$ In the systematic review by Salgado et $\mathrm{al}^{5}$ the authors speculated that the increased morbidity and mortality could be because patients with VRE bacteremia were more likely to receive ineffective therapy. ${ }^{5,6}$ However, our findings suggest that a lack of effective therapy is not the explanation. It should be noted that our systematic review was unable to capture time to effective therapy. Thus, it is possible that patients with VSE bacteremia received effective therapy sooner than patients with VRE bacteremia because VRE may be less likely to be covered by empiric therapy, and effective therapy may only have been administered following a VRE-positive microbiological culture result. ${ }^{6,15,18,19}$

An alternative explanation for the observed increase in mortality risk and LOS could be differences in illness severity or comorbidities between patients with VRE and VSE bacteremia, particularly because patients with VRE bacteremia may have more comorbidities. ${ }^{18,24,28}$ Due to limited reporting of adjusted mortality and morbidity risks among included studies, we were unable to calculate adjusted summary estimates in this systematic review. Thus, the effect of confounding factors on our unadjusted mortality and LOS summary estimates remains unclear. We hypothesize that not adjusting for potential confounders (ie, comorbid conditions and severity of illness) may lead to overestimates of our associations of interest because patients colonized with VRE tend to have more comorbid conditions and more severe illness than patients not colonized with VRE. ${ }^{18}$

However, the earlier systematic review by DiazGranados et al, ${ }^{6}$ which only considered studies controlling for underlying severity of illness, found VRE bacteremia adjusted mortality risk to be greater in comparison to VSE bacteremia.

The worse outcomes associated with VRE bacteremia compared to VSE bacteremia may also be linked to differences in the causative species as there may have been proportionately more patients with E. faecium than E. faecalis in the VRE bacteremia group when compared to the VSE bacteremia group. ${ }^{1,2}$

Our results should be interpreted recognizing the systematic review's limitations. First, studies included within each meta-analysis were non-randomized observational studies, and accordingly, our results reflect association and not causation. Second, as discussed above, only a small number of 


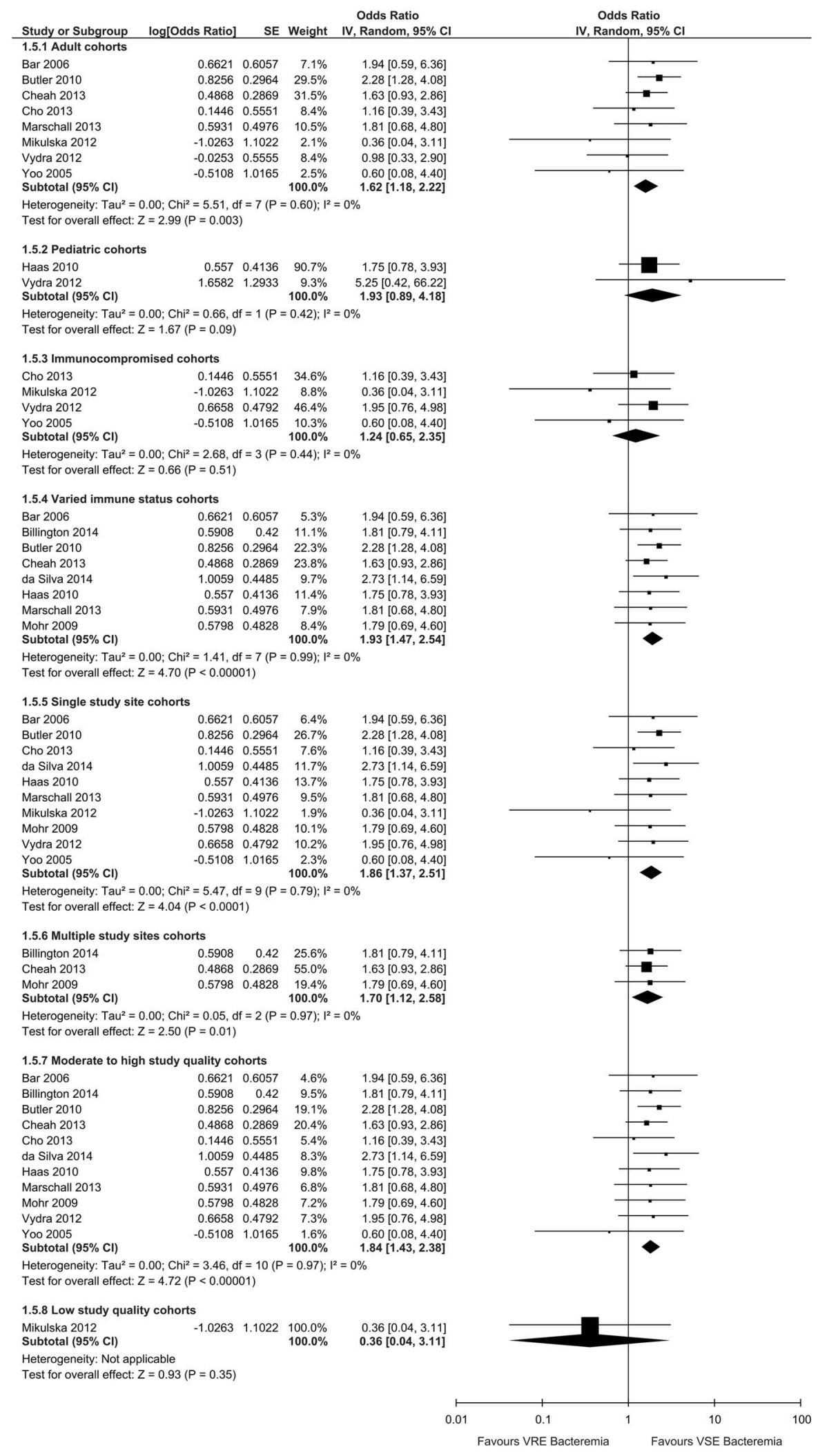

FIGURE 5. Subgroup analysis of VRE and VSE bacteremia un-adjusted in-hospital mortality risk by age, immune status, study site(s), and study quality, for each included cohort study reporting these data. Abbreviations: $95 \%$ CI, 95\% confidence interval; SE, standard error; IV, random, inverse-variance, random-effects method. 


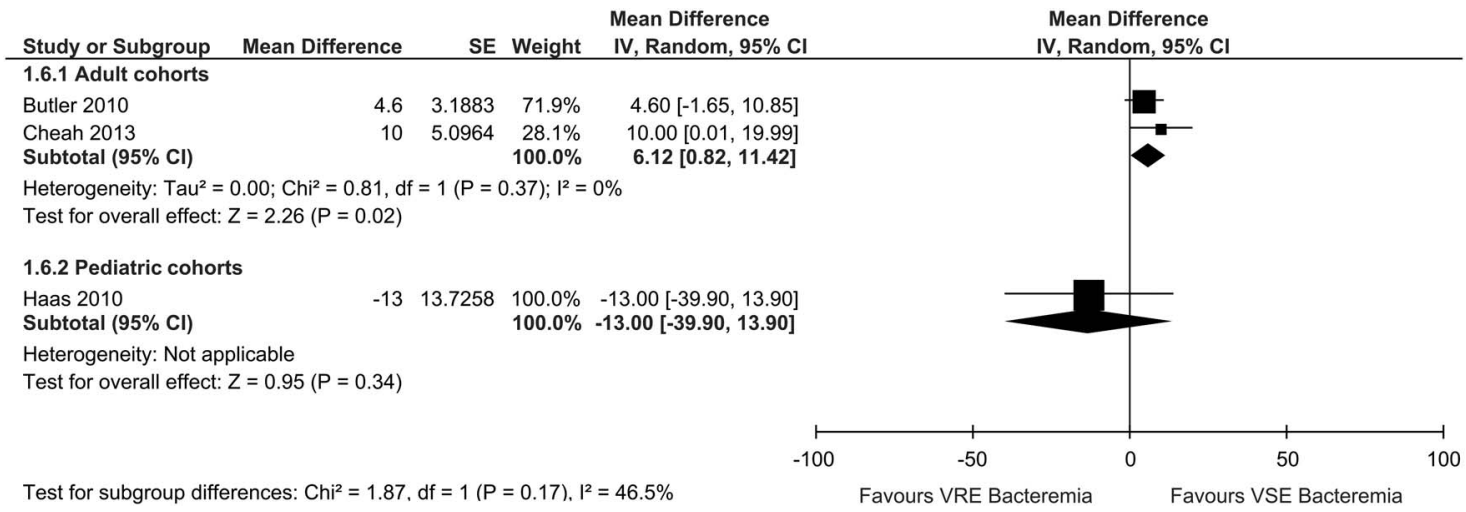

FIGURE 6. Subgroup analysis of VRE and VSE bacteremia hospital LOS by age, for each included cohort study reporting these data. Abbreviations: LOS, length of stay; 95\% CI, 95\% confidence interval; SE, standard error; IV, random, inverse-variance, random-effects method.

TA B LE 2. Assessment of Study Quality, Based on the Newcastle-Ottawa Scale (NOS) Star System

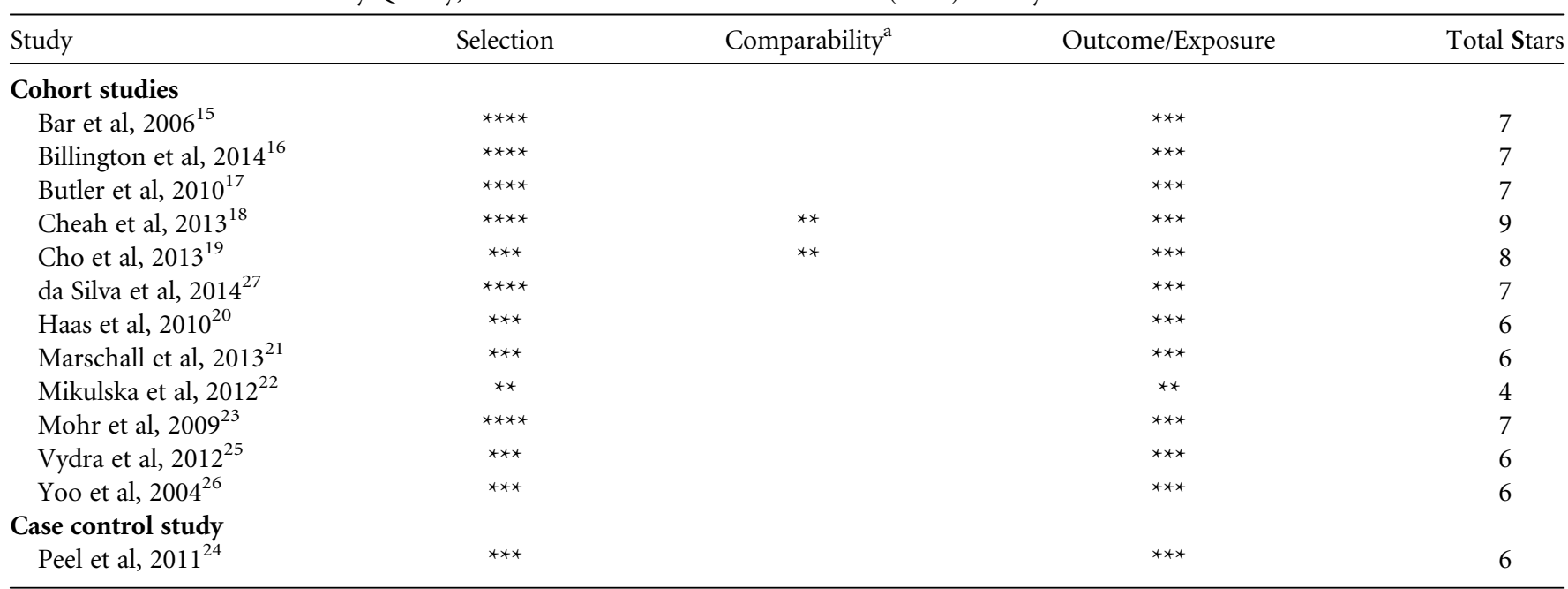

${ }^{a}$ Illness severity and comorbid conditions were selected as the most important factors when assessing comparability.

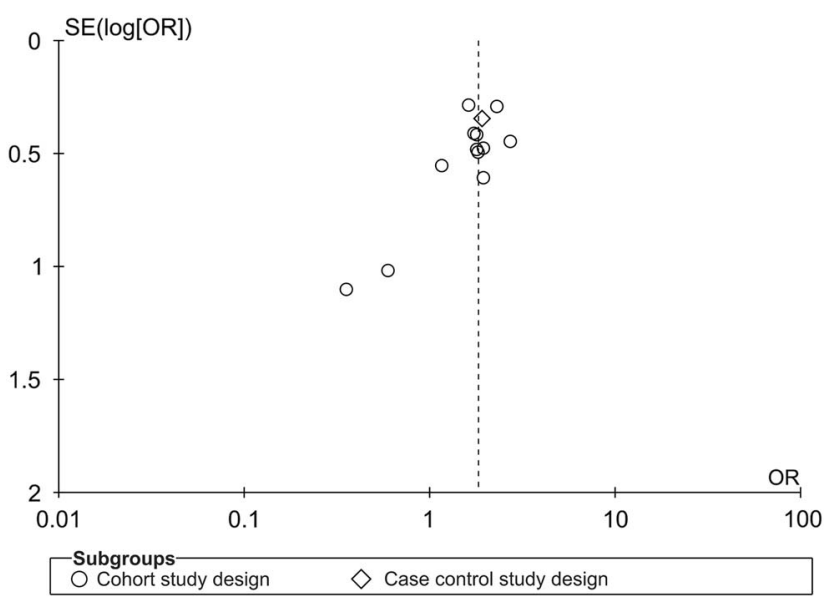

FIgURE 7. Asymmetrical funnel plot of VRE and VSE bacteremia in-hospital mortality effect estimates of all included studies. studies adjusted for potential confounders, and thus confounding may have influenced the investigated associations. Third, our results may be limited by the exclusive review of English language reports published after January 1997, but it is unlikely such language restrictions biased our findings. ${ }^{12}$ Fourth, the majority of studies sampled immunocompromised hospital patient populations, which limited our ability to generalize our findings to all healthcare settings. Last, our funnel plot suggests that there may be publication bias. However, the 2 studies that contributed to this asymmetry had high standard error and odds ratios close to 1 . Thus, if the asymmetry in the funnel plot is due to publication bias, it would bias the results towards the null hypothesis.

We conclude that using the best available evidence, VRE bacteremia remains associated with increased risk of morbidity and mortality when compared with VSE bacteremia in the era of 
effective VRE therapy. Future research is needed to determine whether these results are related to unadjusted differences in the patient populations, differences in treatment effectiveness, or differences in proportions of patients with $E$. faecalis and E. faecium comprising the VRE and VSE bacteremias.

\section{ACKNOW LEDGMENTS}

The authors would like to acknowledge Public Health Ontario, Library Services for their contributions to the development and execution of literature searches referenced in this work.

Financial support: Financial support for this work was provided by Ontario Agency for Health Protection and Promotion (Public Health Ontario).

Potential conflicts of interest: All authors have no potential conflicts of interest to report. The work under consideration for publication was solely supported by funds from Ontario Agency for Health Protection and Promotion (Public Health Ontario) and did not receive third-party funds. All listed authors have no financial relationships with entities in the biomedical arena that could be perceived to influence, or that give the appearance of potentially influencing, the submitted work. Finally, all listed authors have no other relationships or activities that readers could perceive to have influenced, or that give the appearance of potentially influencing, what is written in the submitted work.

Address correspondence to Jennie Johnstone, $\mathrm{MD}, \mathrm{PhD}$, Public Health Ontario, 480 University Avenue, Suite 300, Toronto ON M5G 1V2 (jennie.johnstone@oahpp.ca)

SUPPLEMENTARY MATERIAL

To view supplementary material for this article, please visit http://dx.doi.org/10.1017/ice.2015.228

\section{REFERENCES}

1. Cetinkaya Y, Falk P, Mayhall CG. Vancomycin-resistant enterococci. Clin Microbiol Rev 2000;13:686-707.

2. Murray BE. Vancomycin-resistant enterococcal infections. N Engl J Med 2000;342:710-721.

3. Ramsey AM, Zilberberg MD. Secular trends of hospitalization with vancomycin-resistant Enterococcus infection in the United States, 2000-2006. Infect Control Hosp Epidemiol 2009; 30:184-186.

4. Gravel D, Archibald CP, Pelude L, Mulvey M, Golding G. Antimicrobial resistance surveillance in Canadian hospitals, 2007-2012. Canada Communicable Disease Report CCDR 2014, Volume 40:November 21, 2014. 40 S-40 2; Accessed November 21, 2014.

5. Salgado CD, Farr BM. Outcomes associated with vancomycinresistant enterococci: a meta-analysis. Infect Control Hosp Epidemiol 2003;24:690-698

6. DiazGranados CA, Jernigan JA. Impact of vancomycin resistance on mortality among patients with neutropenia and enterococcal bloodstream infection. J Infect Dis 2005;191:588-595.

7. Erlandson KM, Sun J, Iwen PC, Rupp ME. Impact of the more-potent antibiotics quinupristin-dalfopristin and linezolid on outcome measure of patients with vancomycin-resistant Enterococcus bacteremia. Clin Infect Dis 2008;46:30-36.

8. Balli EP, Venetis CA, Miyakis S. Systematic review and meta-analysis of linezolid versus daptomycin for treatment of vancomycin-resistant enterococcal bacteremia. Antimicrob Agents Chemother 2014;58:734-739.

9. Moher D, Liberati A, Tetzlaff J, Altman DG, PRISMA Group. Preferred reporting items for systematic reviews and meta-analyses: the PRISMA statement. BMJ 2009;339:b2535.

10. Cochrane Handbook for Systematic Reviews of Interventions. Version 5.1.0 ed. The Cochrane Collaboration; 2011.

11. Linden PK. Treatment options for vancomycin-resistant enterococcal infections. Drugs 2002;62:425-441.

12. Moher D, Pham B, Lawson ML, Klassen TP. The inclusion of reports of randomised trials published in languages other than English in systematic reviews. Health Technol Assess 2003; 7:1-90.

13. Wells GA, Shea B, O'Connell D, et al. The Newcastle-Ottawa Scale (NOS) for assessing the quality of nonrandomized studies in meta-analyses. Ottawa hospital Research Institute Website. http://www.ohri.ca/programs/clinical_epidemiology/oxford.asp. Published 2014. Accessed April, 20, 2015.

14. Hozo SP, Djulbegovic B, Hozo I. Estimating the mean and variance from the median, range, and the size of a sample. BMC Med Res Methodol 2005;5:13.

15. Bar K, Wisplinghoff H, Wenzel RP, Bearman GM, Edmond MB. Systemic inflammatory response syndrome in adult patients with nosocomial bloodstream infections due to enterococci. BMC Infect Dis 2006;6:145.

16. Billington EO, Phang SH, Gregson DB, et al. Incidence, risk factors, and outcomes for Enterococcus spp. blood stream infections: a population-based study. Int J Infect Dis 2014;26: 76-82.

17. Butler AM, Olsen MA, Merz LR, et al. Attributable costs of enterococcal bloodstream infections in a nonsurgical hospital cohort. Infect Control Hosp Epidemiol 2010;31:28-35.

18. Cheah AL, Spelman T, Liew D, et al. Enterococcal bacteraemia: factors influencing mortality, length of stay and costs of hospitalization. Clin Microbiol Infect 2013;19:E181-E189.

19. Cho SY, Lee DG, Choi SM, et al. Impact of vancomycin resistance on mortality in neutropenic patients with enterococcal bloodstream infection: a retrospective study. BMC Infect Dis 2013;13:504.

20. Haas EJ, Zaoutis TE, Prasad P, Li M, Coffin SE. Risk factors and outcomes for vancomycin-resistant Enterococcus bloodstream infection in children. Infect Control Hosp Epidemiol 2010;31: 1038-1042.

21. Marschall J, Piccirillo ML, Fraser VJ, Doherty JA, Warren DK. Catheter removal versus retention in the management of catheter-associated enterococcal bloodstream infections. Can J Infect Dis Med Microbiol 2013;24:e83-e87.

22. Mikulska M, Del Bono V, Raiola AM, et al. Enterococcal bloodstream infection after hematopoietic stem cell transplant: experience of a center with a low prevalence of vancomycine8resistant enterococci. Clin Infect Dis 2012;55:1744.

23. Mohr JF, Friedrich LV, Yankelev S, Lamp KC. Daptomycin for the treatment of enterococcal bacteraemia: results from the Cubicin Outcomes Registry and Experience (CORE). Int J Antimicrob Agents 2009;33:543-548.

24. Peel T, Cheng AC, Spelman T, Huysmans M, Spelman D. Differing risk factors for vancomycin-resistant and vancomycinsensitive enterococcal bacteraemia. Clin Microbiol Infect 2012; 18:388-394. 
25. Vydra J, Shanley RM, George I, et al. Enterococcal bacteremia is associated with increased risk of mortality in recipients of allogeneic hematopoietic stem cell transplantation. Clin Infect Dis 2012;55:764-770.

26. Yoo JH, Lee DG, Choi SM, et al. Vancomycin-resistant enterococcal bacteremia in a hematology unit: molecular epidemiology and analysis of clinical course. J Korean Med Sci 2005;20: 169-176.
27. da Silva NS, Muniz VD, Estofolete CF, Furtado GH, Rubio FG. Identification of temporal clusters and risk factors of bacteremia by nosocomial vancomycin-resistant enterococci. Am J Infect Control 2014;42:389-392.

28. Cheah AL, Peel T, Howden BP, et al. Case-case-control study on factors associated with vanB vancomycin-resistant and vancomycin-susceptible enterococcal bacteraemia. BMC Infect Dis 2014;14:353. 